\title{
Genotype and phenotype in Alzheimer's disease ${ }^{\dagger}$
}

\author{
CLIVE HOLMES
}

\begin{abstract}
Background Patients with Alzheimer's disease show a wide variation in clinical phenotype. Genetic research has been largely concerned with the role of mutations or common variants as risk factors for the disease. Do genetic factors also influence clinical phenotype?
\end{abstract}

\begin{abstract}
Aims To examine the evidence that genetic factors influence the clinical expression of the disease in addition to influencing risk.
\end{abstract}

Method A selective review was made of the key literature.

Results Mutations in three genes, coding for amyloid precursor protein, presenilin-I and presenilin-2, and a common variation ( $\varepsilon 4)$ in another gene, APOE, have been shown to lead to an earlier development of the disease. More recently, genetic association and twin studies have suggested a role for genetic factors in the development of other aspects of clinical phenotype, notably the appearance of non-cognitive symptoms.

Conclusions In Alzheimer's disease genetic variation influences a number of aspects of clinical phenotype.

Declaration of interest None.
Early family and twin studies have indicated that a number of genes have an important part to play in the development of Alzheimer's disease. Variation in these genes, including both rare mutations and common polymorphisms, appears to confer increased risk for the development of the disease. However, apparent increased risk may be largely explained by the effects that genetic variation has on the clinical phenotype of Alzheimer's disease, particularly the age at which the disease presents. More recently, it has been proposed that genetic variation may also explain some of the other features of clinical phenotype, including the development of non-cognitive symptoms.

\section{GENES AS RISK FACTORS}

The clearest evidence for a genetic contribution to the aetiology of Alzheimer's disease is the existence of families in whom the disease is transmitted in a clear autosomal dominant pattern. Study of one of these large pedigrees revealed the transmission, from generation to generation, of a single point mutation in the gene for amyloid precursor protein (APP), found on chromosome 21 in family members developing early-onset Alzheimer's disease. Further studies of these families led to the discovery of other point mutations in the same gene and later the presence of mutations in two other genes, encoding presenilin-1 (PS-1) and presenilin-2 (PS-2), found on chromosomes 14 and 1 , respectively. To date, 12 Alzheimer's disease-related mutations have been discovered in the APP gene, 69 mutations in the PS- 1 gene and 5 mutations in the PS- 2 gene. Significantly, research suggests that the different mutations in these three genes lead to a common result, an increase in $\beta$ A4 peptide fragment of APP which forms the core of the neuritic plaques found in the brains of people with Alzheimer's disease.

While these findings are of immense importance in terms of elucidating the biological pathogenesis of Alzheimer's diease, it is important to recognise that these three causal genes may only account for $30-50 \%$ of all autosomal dominant early-onset cases, or around $10 \%$ of familial early-onset cases (Cruts et al, 1998). Thus, given that in the majority of cases (>95\%) Alzheimer's disease occurs after the age of 60 years, it is clear that these genetic mutations make only a small contribution to the risk of developing the disease in general.

The inheritance of late-onset Alzheimer's disease is more complex than that of the early-onset form. In cross-sectional studies the presence of a positive family history of late-onset Alzheimer's disease is a consistent risk factor for subjects with the late-onset form, but a clear autosomal dominant pattern of inheritance is rare. Both twin and family studies suggest a polygenic multifactorial mode of inheritance and as a consequence the elucidation of genetic risk factors for late-onset Alzheimer's disease requires different methods from those used in the early-onset disease, with its simple Mendelian patterns of inheritance. Association studies have so far dominated the genetics of late-onset Alzheimer's disease. Such studies look for variation in the frequency of an allele in samples of unrelated patients with late-onset Alzheimer's disease compared with unrelated control subjects. These studies have resulted in a number of candidate genes being proposed as having importance as risk factors for late-onset Alzheimer's disease, and include associations with variation in genes encoding the very lowdensity lipoprotein (VLDL) receptor, PS-1 (an intronic mutation not associated with early-onset Alzheimer's disease), butyrylcholinesterase, angiotensin-1 converting enzyme, $\alpha_{1}$-antichymotrypsin, the human leucocyte antigen (HLA) complex, $\alpha_{2}$-macroglobulin, the low-density lipoprotein-like receptor, the serotonin transporter and interleukin-1. Typically, interest in these candidates has diminished as independent replication has not been forthcoming, and little evidence now remains for an important role for genetic variation in the VLDL receptor, PS- 1 or $\alpha_{1}$-antichymotrypsin genes in the development of late-onset Alzheimer's disease. The other candidate genes still have their advocates and remain hotly disputed. 
More recently, the results of a number of linkage studies have become available. The methods used for linkage studies in complex non-Mendelian diseases such as late-onset Alzheimer's disease are less powerful than those used for the earlyonset form. However, such studies can reveal areas of interest in the genome that might later be explored by association studies. A complete genomic screen in a series of multiplex families affected with late-onset Alzheimer's disease has revealed five regions of interest on chromosomes 4, 6, 12, 19 and 20. Excluding the effects of the APOE4 locus, the maximum log likelihood ratio (lod) score in this study occurred in a region located on the short arm of chromosome 12 (Pericak-Vance et al, 1997). However, two subsequent studies following similar methods (Blacker et al, 1998; Wu et al, 1998) were unable to confirm linkage to this area. A large sibpair study (Kehoe et al, 1999) has revealed many possible loci showing linkage to lateonset Alzheimer's disease. These include loci on chromosomes $1,5,9,10,12,14$, 19 (close to $A P O E$ ) and 21 (close to the APP gene). Currently, the main focus of attention is chromosome 10 , with two independent studies showing evidence of a susceptibility locus in the same region (Ertekin-Taner et al, 2000; Myers et al, 2000). However, it is clear that a large number of loci are implicated and that there is some disagreement between studies in the predicted areas of interest. These findings demonstrate some of the limitations of this approach, but also serve to emphasise the polygenic nature of lateonset Alzheimer's disease. Further exploration of these areas by association studies the positional candidate approach - is likely to dominate the genetics of Alzheimer's disease in the future.

Of the genetic risk factors that have been advocated as having a role in the development of late-onset Alzheimer's disease, the least controversial remains the increased risk associated with possession of one or more copies of the APOE4 allele, coding for the apolipoprotein $\mathrm{E}$ (ApoE) variant $\varepsilon 4$. Significantly, the importance of APOE4 was determined using a positional candidate gene approach. Since this finding, other studies (Artiga et al, 1998; Bullido et al, 1998; Lambert et al, 1998) have also suggested an association between a number of polymorphisms within the transcriptional regulatory region of the APOE gene and late-onset Alzheimer's disease that is independent of ApoE $\varepsilon 4$ status. Based on these data, it was suggested that there are two independent mechanisms by which risk of late-onset Alzheimer's disease can be modified by the APOE gene: first, by variations in the coding regions, which alter the functional properties of $A P O E$; and second, by the presence of promoter variants which result in quantitative differences in $A P O E$ expression (Artiga et al, 1998). However, this hypothesis is controversial, and more recent studies (Helisalmi et al, 1999; Zurutuza $e t$ al, 2000) have failed to support the independence of $A P O E$ promoter variants in the development of Alzheimer's disease.

\section{GENETIC INFLUENCES ON CLINICAL PHENOT YPE}

\section{Age of onset}

Families carrying mutations in the APP gene have ages of onset largely within the range 40-65 years. To date, no APP mutation carrier has been reported to be unaffected beyond the age of 67 years. Families carrying mutations in the PS- 1 gene have the earliest ages of onset, which fall largely within the range 35-55 years, although one such family member has recently been recorded with onset at 24 years (Wisniewski et al, 1998). These mutations are largely associated with early-onset familial Alzheimer's disease, but at least two of these mutations (Ala79Val and His163Tyr) have been observed with lateonset Alzheimer's disease and may be non-penetrant (Cruts et al, 1998). Families carrying mutations in the PS- 2 gene have ages of onset largely within the range 40 70 years and thus show some overlap with late-onset Alzheimer's disease. Penetrance is high but may not be $100 \%$, with at least two reported cases of non-penetrance over the age of 80 years (Bird et al, 1996).

The familial influence on age of onset in late-onset Alzheimer's disease may be substantial. Thus, one study of affected sib pairs suggests that shared familial effects may account for $40 \%$ of the variance (Tunstall et al, 2000). Indeed, studies have shown that in the late-onset disease the age of onset is approximately 6 years earlier in individuals who carry two copies of the APOE4 allele compared with non-carriers. This has led to the suggestion that the APOE4 allele is a risk factor for Alzheimer's disease mainly because in old age Alzheimer's disease and death are competing risks. Any factor leading to an earlier age of onset of Alzheimer's disease in the elderly (and hence the development of the disease prior to death) will be associated with Alzheimer's disease. Evidence confirming this hypothesis has come from a large study of approximately 5000 individuals (Meyer et al, 1998). This study showed a clear decline in relative risk of developing late-onset Alzheimer's disease in all subjects with increasing age but with a clear plateau beyond which no further new cases of the disease were reported. The age at which this plateau was reached was earlier in carriers of the APOE4 allele. Thus, it appears clear that the APOE4 allele has its predominant effect by determining when, but not if, an individual develops late-onset Alzheimer's disease (Meyer et al, 1998).

\section{Non-cognitive features}

If genetic risk factors for Alzheimer's disease are associated with non-cognitive symptoms, then one might anticipate that subjects with a family history of the disease have an increased frequency of these symptoms. Likewise, one would expect an association between known genetic risk factors for Alzheimer's disease, including the genes for APP, PS-1, PS-2 and the ApoE $\varepsilon 4$ allele, and these symptoms in Alzheimer's disease.

In fact, there appears to be little consistent evidence of an association between either a positive family history of Alzheimer's disease or variation in known candidate genes and an increase in the frequency of non-cognitive symptoms occurring for the first time within the disease. It thus seems unlikely that major genetic risk factors for the development of late-onset Alzheimer's disease have a substantial role to play in the development of non-cognitive symptoms within the late-onset disease. However, this does not mean that these symptoms have no genetic basis, since genes may influence the phenotypic expression of the disease without being involved directly in its aetiology. Indeed, early studies have shown evidence of an association between a positive family history of depressive illness and an increase in the frequency of depressive symptoms occurring for the first time within Alzheimer's disease (Pearlson et al, 1990). These early findings are supported by a sib-pair study (Tunstall et al, 2000) showing that familial factors have a role in the development of depressive illness in lateonset Alzheimer's disease. In addition, this 
small study also suggests a role for familial factors in a wide variety of noncognitive symptoms, including agitation, aggression and psychosis.

A more direct approach has also been adopted whereby common genetic polymorphisms previously showing associations with other neuropsychiatric conditions have been found to be associated with non-cognitive symptoms in late-onset Alzheimer's disease. For example, one study (Holmes et al, 1998) of people with late-onset Alzheimer's disease found an association between common polymorphic variations in the $5-\mathrm{HT}_{2 \mathrm{~A}}$ and $5-\mathrm{HT}_{2 \mathrm{C}}$ serotonin receptor genes $(102-\mathrm{T} / \mathrm{C}$ and Cys23Ser polymorphisms, respectively), previously implicated in schizophrenia and bipolar affective disorder, with psychotic symptoms in Alzheimer's disease. Associations were found between the presence of the C102 allele and the presence of visual and auditory hallucinations and between the presence of the Ser23 allele and visual hallucinations. Another study (Sweet et al, 1998) has found evidence to suggest an association of psychosis and aggression in patients with Alzheimer's disease who carry common polymorphisms in the dopamine receptor genes DRD1 and DRD3. These studies need to be replicated but they suggest that common receptor polymorphisms may have a role in the genesis of psychotic or other non-cognitive symptoms that are not implicated as genetic risk factors for late-onset Alzheimer's disease.

\section{Cognitive decline}

No study to date has looked at rates of decline in carriers of APP, PS-1 and PS-2 mutations, but patients with late-onset Alzheimer's disease who have a positive family history of Alzheimer's disease show no evidence of increased rates of decline. The majority of studies have also failed to show any relationships between possession of the APOE4 allele and increased rates of decline. This lack of association is interesting since it implies that genetic factors may determine only when the disease starts, not the rate of degeneration. Clearly, caution is required when interpreting a negative finding and it may be that associations will always be difficult to establish because of the large number of confounding factors which may influence cognitive deterioration as well as difficulties with the use of psychometric scales over short periods. Indeed, a study by Craft et al (1998) has

\section{CLINICAL IMPLICATIONS}

- A number of genes have been implicated as risk factors for the development of Alzheimer's disease.

- Variation in some of these genes appears to have a major effect in determining when - not if - the disease will develop.

- Other aspects of clinical phenotype, notably non-cognitive symptoms, are also influenced by genetic variation.

\section{LIMITATIONS}

- A number of other risk genes have yet to be identified.

- The influence of these unknown genes on clinical phenotype is as yet undetermined.

- The influence of genetic variation on non-cognitive symptoms is relatively unexplored.

CLIVE HOLMES, MRCPsych, Thornhill Research Unit, University of Southampton, Moorgreen Hospital, Botley Road, West End, Southampton SO30 3JB, UK

(First received 16 May 2000, final revision 14 May 200I, accepted 23 May 200I)

suggested that there is an increased rate of cognitive decline in APOE4 carriers, which may be detectable by using a long followup period.

\section{Duration}

The mean duration of illness in families with PS-1 mutations is significantly shorter (range 5.8-6.8 years) than in families with both PS-2 (range 4.4-10.8 years) and APP mutations (range 9.0-16 years), reflecting the severity of PS-1-associated Alzheimer's disease. Studies of late-onset Alzheimer's disease suggest that the duration of illness tends to be longer in people who have a positive family history or in carriers of the APOE4 allele. However, it has since been shown that this relationship no longer holds true once the confounding effects of age of onset have been taken into account. Thus, in late-onset Alzheimer's disease, increased survival is more directly related to an earlier age of onset, which is influenced by a number of factors that are not exclusively genetic.

\section{REFERENCES}

Artiga, M. J., Bullido, M. J., Frank, A., et al (1998) Risk for Alzheimer's disease correlates with transcriptional activity of the APOE gene. Human Molecular Genetics, 7, 1887-1892.

Bird, T. D., Levy-Lahad, E., Poorkaj, P., et al (1996) Wide range in age of onset for chromosome l-related familial Alzheimer's disease. Annals of Neurology, 40, 932-936

Blacker, D., Wilcox, M. A., Laird, N. M., et al (1998) Alpha-2 macroglobulin is genetically associated with Alzheimer's disease. Nature Genetics, 19, 357-360.

Bullido, M. J., Artiga, M. J., Recuero, M., et al (1998) A polymorphism in the regulatory region of APOE associated with risk for Alzheimer's dementia. Nature Genetics, 18, 69-7|.

Craft, S., Teri, L., Edland, S. D., et al (1998) Accelerated decline in apolipoprotein E-epsilon4 homozygotes with Alzheimer's disease. Neurology, 5I, 149-153.

Cruts, M., van Duijn, C. M., Backhovens, H., et al (1998) Estimation of the genetic contribution of presenilin-I and -2 mutations in a population-based study of presenile Alzheimer disease. Human Molecular Genetics, 7, 43-5I.

Ertekin-Taner, N., Graff-Radford, N., Younkin, L., et al (2000) Linkage of plasma $A \beta 42$ to a quantitative locus on chromosome 10 in late onset Alzheimer's disease pedigrees. Science, 290, 2303-2304.

Helisalmi, S., Hiltunen, M., Valonen, M., et al (1999) Promoter polymorphism (-49IA/T) in the APOE gene of Finnish Alzheimer's disease patients and control individuals. Journal of Neurology, 246, 821-824. 
Holmes, C., Arranz, M. J., Powell, J. F., et al (1998) 5-HT2 A and 5-HT2C receptor polymorphisms and psychopathology in late onset Alzheimer's disease. Human Molecular Genetics, 7, 1507-1509.

Kehoe, P., Wavrant-DeVrieze, F., Crook, R., et al (1999) A full genome scan for late onset Alzheimer's disease. Human Molecular Genetics, 8, 237-245.

Lambert, J. C., Berr, C., Pasquier, F., et al (1998) Pronounced impact of Thl/E47cs mutation compared with -49। AT mutation on neural APOE gene expression and risk of developing Alzheimer's disease. Human Molecular Genetics, 7, I5II-1516.

Meyer, M. R., Tschanz, J.T., Norton, M. C., et al (1998) APOE genotype predicts when - not

whether - one is predisposed to develop Alzheimer disease. Nature Genetics, 19, 321-322.
Myers, A., Holmans, P., Marshall, H., et al (2000) Susceptibility locus for Alzheimer's disease on chromosome I0. Science, 290, 2304-2305.

Pearlson, G. D., Ross, C. A., Lohr, W. D., et al (1990) Association between family history of affective disorder and the depressive syndrome of Alzheimer's disease. American Journal of Psychiatry, 147, 452-456.

Pericak-Vance, M. A., Bass, M. P., Yamaoka, L. H., et al (1997) Complete genomic screen in late-onset familial Alzheimer disease. Evidence for a new locus on chromosome 12. JAMA, 278, 1237-1241.

Sweet, R. A., Nimgaonkar, V. L., Kamboh, M. I., et a (1998) Dopamine receptor genetic variation, psychosis, and aggression in Alzheimer's disease. Archives of Neurology, 55, 1335-1340.

Tunstall, N., Owen, M. J., Williams, J., et al (2000) Familial influence on variation in age of onset and behavioural phenotype in Alzheimer's disease. British Journal of Psychiatry, 176, I56-159.

Wisniewski, T., Dowjat, W. K., Buxbaum, J. D., et a (1998) A novel Polish presenilin-2 mutation (PII7L) is associated with familial Alzheimer's disease and leads to death as early as the age of 28 years. Neuroreport, 9 2|7-22|

Wu, W. S., Holmans, P., Wavrant-DeVrieze, F., et al (1998) Genetic studies on chromosome 12 in late-onset Alzheimer disease. JAMA, 280, 619-622.

Zurutuza, L., Verpillat, P., Raux, G., et al (2000) APOE promoter polymorphisms do not confer independent risk for Alzheimer's Disease in a French population. European Journal of Human Genetics, 8 $713-716$ 